\title{
Avalanches in a granular stick-slip experiment: detection using wavelets
}

\author{
Aghil Abed Zadeh ${ }^{1, \star}$, Jonathan Barés ${ }^{2, \star \star}$, and Robert P. Behringer ${ }^{1, \star \star \star}$ \\ ${ }^{1}$ Duke University, Durham, NC, 27708, USA \\ ${ }^{2}$ Laboratoire de Mécanique et Génie Civil, Université de Montpellier, CNRS, Montpellier, France
}

\begin{abstract}
Avalanches have been experimentally investigated in a wide range of physical systems from granular physics to friction. Here, we measure and detect avalanches in a 2D granular stick-slip experiment. We discuss the conventional way of signal processing for avalanche extraction and how statistics depend on several parameters that are chosen in the analysis process. Then, we introduce another way of detecting avalanches using wavelet transformations that can be applied in many other systems. We show that by using this method and measuring Lipschitz exponents, we can intelligently detect noise in a signal, which leads to a better avalanche extraction and more reliable avalanche statistics.
\end{abstract}

\section{Introduction}

Many systems, under slow loading, exhibit intermittent and discrete events of broad sizes, known as crackling noise $[1,2]$. This behavior has been experimentally investigated in a wide range of physical systems [3-5] from earthquakes in seismic activities to Barkhausen noise in ferromagnets $[6,7]$. Slowly sheared granular media are examples of these systems. They respond to shear stress with discrete slip avalanches, also called stick-slip events [811]. The avalanches statistics of these systems and their universal and non-universal quantities are key properties of these systems. In this paper, we introduce a method for avalanche detection based on wavelet transformation to extract more reliable statistics. This method can be used for many other systems for a better avalanche detection.

As shown in figure 1, to observe the physics of stickslip for slowly sheared granular materials, we have developed a novel apparatus, which is capable of detecting avalanches both at the global and local scales. In our experiments, a constant speed stage pulls a frictional slider of length $25 \mathrm{~cm}$ which rests on a vertical bed of bi-disperse cylindrical photo-elastic particles with diameteres of 0.4 $\mathrm{cm}$ and $0.5 \mathrm{~cm}$ (number ratio of 2.7) in a 2D granular system. The verticle walls are low frictional to reduce the efect of boundary friction. The slider is designed to have very high friction with the top layer of granular media. The stage is connected to the slider by a spring, the stiffness of which can be tuned. We measure the force on the spring by a force sensor attached to it, which provides a global measure of shear stress. As we want to fix the system stiffness and pressure at a certain chosen value; it is not possible to modify the device to force the slider to remain horizontal.

\footnotetext{
^e-mail: aghil.abed.zadeh@duke.edu

$\star \star$ e-mail: jonathan.bares@umontpellier.fr

$\star \star \star$ e-mail: bob@phy.duke.edu
}

However, to make sure the slider always stay in the same inclination; we have calibrated its wait and initial angle so that it may tilt only few degrees. That may add some noise to the system; however, we think is not significant. The photo-elastic response also provides us a measure of local stress; however, in this paper, we focus on the global response of the system.

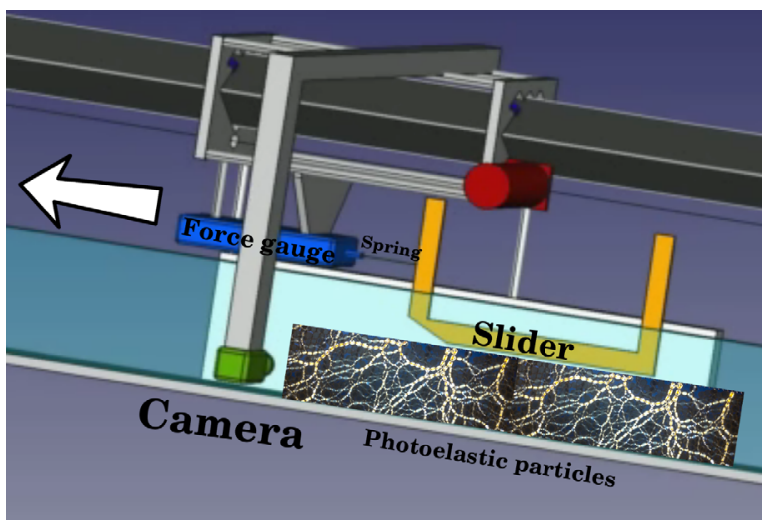

Figure 1. Side view of apparatus. A bi-disperse photo-elastic granular media is sheared by a slider that is moved by the force gauge connected to a spring. The slider exhibits a stick and slip dynamics. The force on the slider is recorded via a force gauge and the internal structure and stress is imaged via high speed cameras (not treated in this paper).

For a given pulling stage speed $v$, spring stiffness $k$, and granular bed height $l$, the evolution of the force $F$, applied to the slider is measured with a frequency of $100 \mathrm{~Hz}$ in our experiment. To quantify the global avalanche statistics based on $F(t)$, we use specific signal processing. In experimental data, noise is unavoidable, but the way noise is detected or reduced can be crucial to quantitative re- 


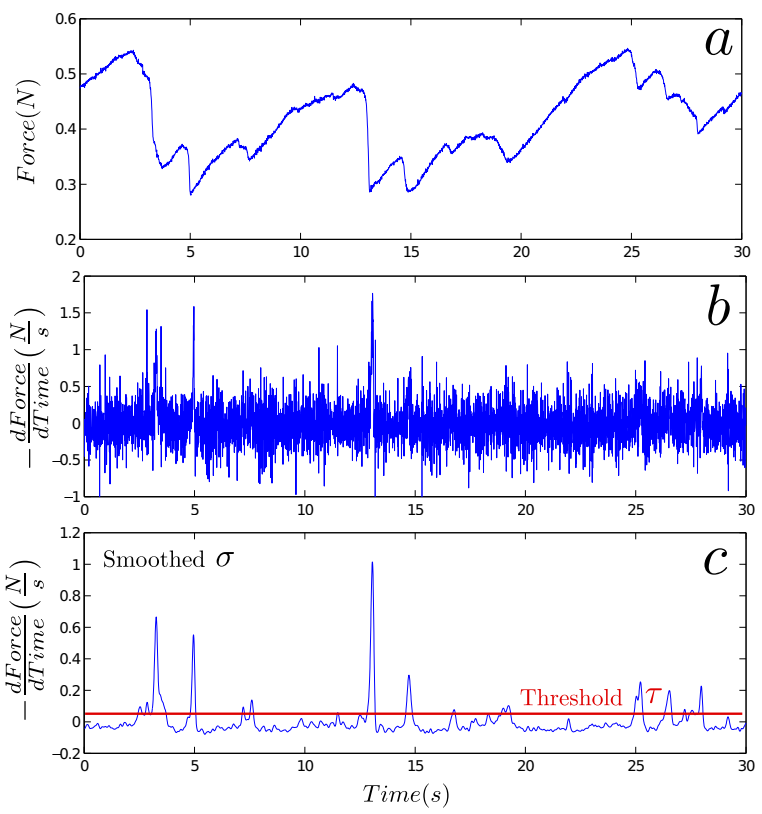

Figure 2. a) A typical force gauge signal $F(t)$ from the experiment with spring stiffness of $k=70 \mathrm{~N} / \mathrm{m}$ and stage speed of $v=1 \mathrm{~mm} / \mathrm{s}$ acquired at $100 \mathrm{~Hz}$. b) Point to point derivative of force signal. The signal-to-noise ratio decreases dramatically when differentiated. c) The smoothed derivative of force signal. Smoothing is applied by a convolution of part b with a Gaussian function of standard deviation $\sigma=0.05 \mathrm{~s}$. The threshold $\tau$ (red line) determines the beginnings and endings of avalanches.

sults. Conventionally, these noise spikes are removed using Fourier transform domain filters, including averaging over many data points. The latter is a low-pass filter in Fourier transform domain and increases the signalto-noise ratio. However, the trade-off is loosing the edge information. Moreover, if the duration of an event in a signal is of the order of the duration of a noise spike, the event may break into several events and make them hardly distinguishable; or some noise spikes may be detected as events. These flaws can be reduced by applying a wavelet transform domain filter that selectively distinguishes noise spikes from events.

In the first part of this paper, we summarize a conventional way of avalanche detection in a typical signal of our experiment. We discuss how several chosen parameters in the signal analysis process can affect the statistics. Then, we introduce a wavelet transform domain filter and how to selectively detect noise spikes locally using Lipschitz exponents [12], without loosing other signal information. This method may provide a tool for a better avalanche extraction in many other experimental systems.

\section{Conventional method}

The slow shearing of a granular materials leads to intermittent slow loading and fast unloading of the medium that is called an avalanche. Figure 2 a shows a typical signal $F(t)$ from the force gauge. The fast drops in $F$ (like at time $t=13 \mathrm{~s}$ ) correspond to slip avalanches of the slider and similar behavior in observables have been detected in many physical systems $[5,8,13,14]$. Hence, to detect an avalanche, we need to find where the slope of the force is negative. However, if we use point to point derivatives, as shown in figure $2 b$, the presence of noise may make it impossible to find the avalanches. To overcome this problem conventionally, as depicted in figure $2 \mathrm{c}$, the signal is smoothed and then a threshold $\tau$ is chosen for finding where it crosses the smoothed data. This defines the beginnings and endings of avalanches. To smooth the signal from our data, we applied a convolution as presented in equation 1 , in which $h$ is the derivative of the force signal $F$ and $g$ is a normalized discrete Gaussian function. This method requires two variables, the standard deviation of the smoothing function, $\sigma$, and the threshold, $\tau$. The statistics of avalanches vary by changing these two variables.

$$
\begin{gathered}
(h * g)[m]=\sum_{t=1}^{n} f[t] g[m-t] \\
g[t]=\frac{1}{\sigma \sqrt{2 \pi}} \exp \left(-\frac{t^{2}}{2 \sigma^{2}}\right)
\end{gathered}
$$

Figure 3 shows the probability density function of slip sizes for different values of the threshold $\tau$ and standard deviation $\sigma$ ( as in fig.2c). The slip size is the displacement of the slider during an avalanche, which is calculated using the force signal $\Delta x=\Delta F / k$, in which $k$ is the stiffness of the spring. As shown in the figure, the probability density function (PDF) is affected by $\tau$ and $\sigma$. Indeed, both the exponent and the non-universal quantities such as lower cut-offs and prefactors depend on these two parameters. As large avalanches are easier to detect and less affected by low-pass filters, the statistics of them do not change dramatically by changing these two variables. However, small avalanches statistics are more affected by these parameters as they contain more high frequency information. This variation can also be seen in other avalanche properties such as the average shape of avalanches (not shown here).

\section{Wavelet transformation method}

Wavelet transforms have been widely used in signal processing in computer science for detection of different types of singularities in a signal [12]. A wavelet transformation of a signal is simply its convolution with a function $g$ as in equation 1 . The convoluting wavelet that we use here is proportional to the first derivative of a Gaussian function, as in equation 3 , which is a widely used wavelet for edge detection problems [12].

$$
g[t]=\frac{t}{\sigma^{2} \sqrt{2 \pi}} \exp \left(-\frac{t^{2}}{2 \sigma^{2}}\right)
$$

Each local maximum in the signal wavelet transformation cooresponds to a "singularity". The dependence of the local maxima on $\sigma$ determines the type of the singularity [12]. Typical noise is associated with a back and forth 

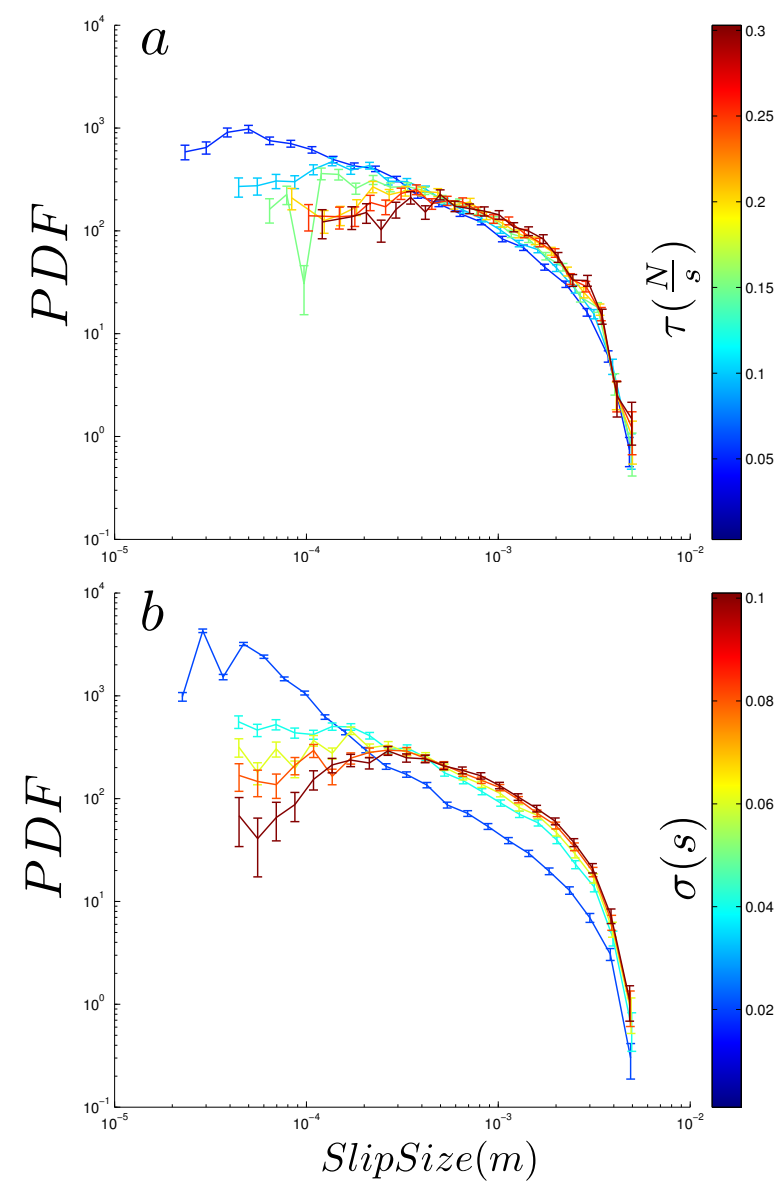

Figure 3. Probability density functions (PDF) of slip sizes during avalanches for: a) Several different values of threshold $\tau$ (for fixed $\sigma=0.05$ ) and b) Several different values of standard deviation $\sigma$ (for fixed $\tau=0.1$ ). These two parameters affect the statistical measurements of the force signal.

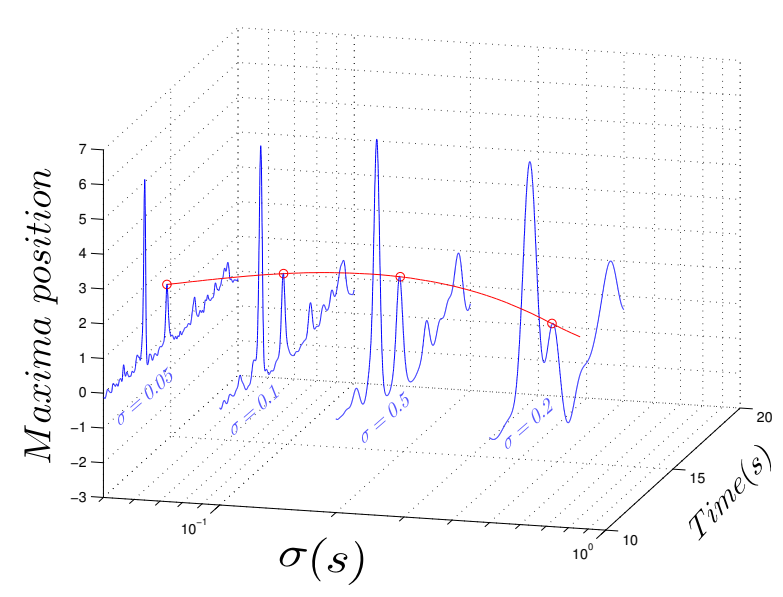

Figure 4. Wavelet transformation of the force signal $F$ of figure $2 \mathrm{a}$ with the wavelet given in equation 3 as a function of $\sigma$. One of local maxima is marked by red circles. We track the position of such local maxima to detect whether it corresponds to an avalanche or not. The avalanche detection is based on the evolution of the maxima position vs $\sigma$.

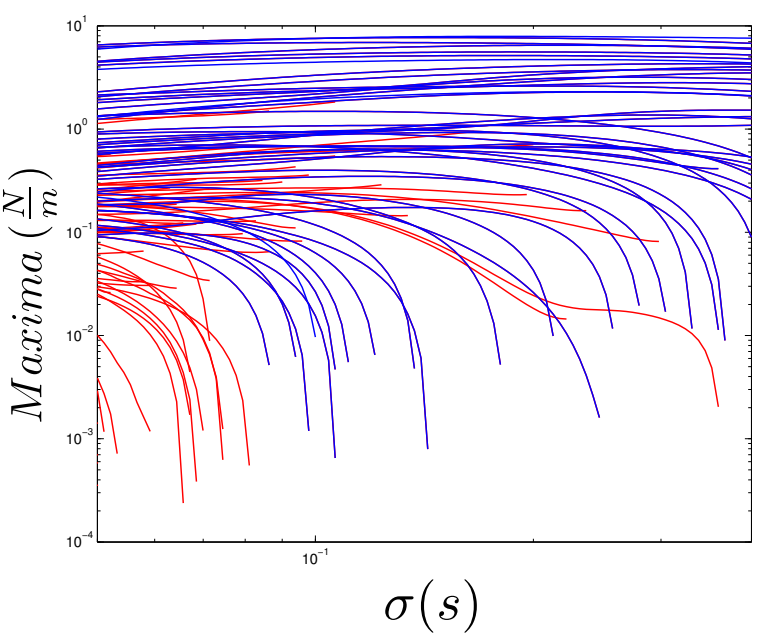

Figure 5. Position of the local maxima (as in fig.4) values as a function of $\sigma$ for a typical force signal $F$. Maxima that are detected to belong to avalanches are marked blue. See text for more details.

peak which resembles a Dirac delta function, whereas, avalanches are sharp unique variations (slow loading and rapid unloading), which resemble step function. This difference can be captured by well chosen wavelets. Figure 4 depicts the wavelet transformation of the force signal $F$ (of fig 2a) as a function of $\sigma$. We track each local maximum, one of which is marked red in figure, vs. $\sigma$ to detect the singularity type.

The evolution of local maxima vs. $\sigma$ for the figure $2 \mathrm{a}$ is shown in figure 5 . Each line in figure 5 belongs to a local maximum. The slope of each line is called a Lipschitz exponent of the singularity cooresponding with the maximum of the signal derivative. This exponent is non-negative for an edge function and negative for a Dirac delta function [12]. Hence, it is also non-negative for a singularity corresponding to avalanche events in our case, and negative for those of noise. Moreover, to detect the avalanches more precisely,we need to have non-negative Lipschitz exponent and a clear exponential decaying tail. By using the Lipschitz exponent and detecting the decaying tail, we can extract the maxima that correspond to the avalanches, which are marked blue in figure 5.

This wavelet transformation method can improve on avalanche detection over the conventional methods, without losing high frequency information. It also provides a better detection of small avalanches. Figure 6 shows the detected avalanches in a force signal $F$. The avalanches are marked as blue and each avalanche is a connected set of blue points in the figure. Using this method, we can then calculate the probability density function of avalanche sizes and other avalanche statistics. Figure 7 shows the PDF of avalanche slip size for instance. This figure is calculated using several thousand detected avalanches for a spring stiffness of $k=70 \mathrm{~N} / \mathrm{m}$ and stage speed of $v=1 \mathrm{~mm} / \mathrm{s}$. The distribution obeys a power-law for about two decades with a clear lower cut-off and an exponen- 


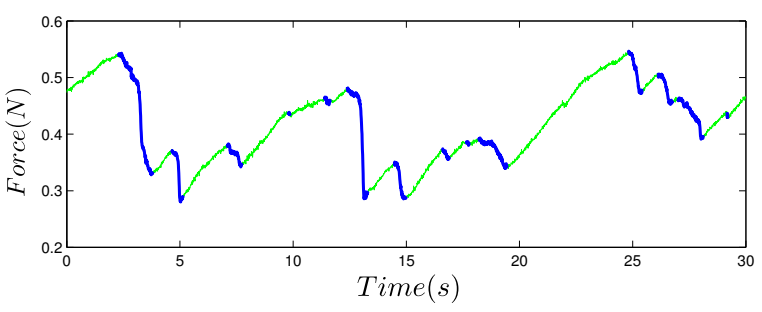

Figure 6. The detected avalanches in a force signal sample using the wavelet transformation method. Avalanches are marked blue. This method has the advantage of better detection of small avalanches and ignoring the noise spikes occurring during avalanches. These noise spikes can break the avalanches into several ones in the conventional method.

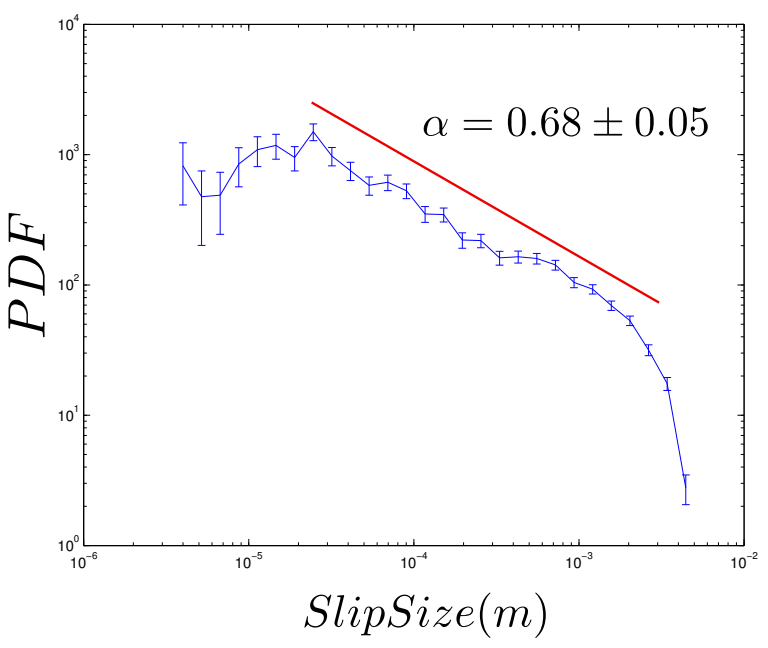

Figure 7. Probability density functions (PDF) of slip sizes during avalanches, detected by the wavelet transformation method. The PDF obeys a power-law for more number of decades compared to conventional method (fig 3), as a result of avalanche detection using wavelets.

tial upper cut-off at slip size $2 \times 10^{-5} \mathrm{~m}$ and $2 \times 10^{-3} \mathrm{~m}$ respectively. Because of better detection of avalanches, the wavelet transformations method provides a smaller lower cut-off and larger number of power-law decades, compared to the conventional method (fig 3). This local noise detection and removal provides a more reliable exponent calculation in the PDF of avalanche sizes over the conventional method, which usually uses global smoothing of the data.

\section{Conclusion}

Our experimental apparatus enables us to study the avalanches in a granular stick-slip experiment. The slow shear of granular system is followed by intermittent fast unloading of shear stress that is measured by a force signal. This force signal is conventionally smoothed (low-pass filter) to increase the signal-to-noise ratio for avalanche detection. However, this conventional method changes the statistics of avalanches as it removes all low frequency information. We introduced a smarter way of avalanche extraction from measured quantities using wavelet transformation. Similar methods have been tested in many other problems and here it provides a better avalanche detection and consequently more reliable statistics, by detecting the noise locally in the signal. The method introduced here can be applied in many other systems for a more precise avalanche extraction.

\section{Acknowledgements}

We acknowledge support from NSF-DMS-1248071, NASA Grant NNX15AD38G, and William M. Keck Foundation.

\section{References}

[1] J.P. Sethna, K.A. Dahmen, C.R. Myers, Nature 410, $242(2001)$

[2] R. Dickman, M.A. Munoz, A. Vespignani, S. Zapperi, Brazilian Journal of Physics 30, 27 (2000)

[3] A. Kabla, J. Scheibert, G. Debregeas, Journal of Fluid Mechanics 587, 45 (2007)

[4] R. Planet, S. Santucci, J. Ortín, Journal of Contaminant Hydrology 120-121, 157 (2011), reactive Transport in the Subsurface: Mixing, Spreading and Reaction in Heterogeneous Media

[5] J. Barés, M.L. Hattali, D. Dalmas, D. Bonamy, Physical Review Letters 113, 264301 (2015)

[6] O. Perkovic, K.A. Dahmen, S.P. Sethna, Physical Review Letters 75, 4528 (1995)

[7] R.A. White, K.A. Dahmen, Physical Review Letters 91, 085702 (2003)

[8] D. Howell, R.P. Behringer, C. Veje, Physical Review Letter 82, 5241 (1999)

[9] F. Dalton, D. Corcoran, Physical Review E 63, 061312 (2001)

[10] K.E. Daniels, N.W. Hayman, Journal of Geophysical Research: Solid Earth 113 (2008)

[11] M. Otsuki, H. Hayakawa, Physical Review E 90, 042202 (2014)

[12] S. Mallat, W.L. Hwang, IEEE transactions on information theory 38, 617 (1992)

[13] E. Aharonov, D. Sparks, Journal of Geophysical Research: Solid Earth 109, n/a (2004)

[14] A. Petri, A. Baldassarri, F. Dalton, G. Pontuale, L. Pietronero, S. Zapperi, The European Physical Journal B 64, 531 (2008) 